(c) 2016 IEEE. Personal use of this material is permitted. Permission from IEEE must be obtained for all other uses, in any current or future media, including reprinting/republishing this material for advertising or promotional purposes, creating new collective works, for resale or redistribution to servers or lists, or reuse of any copyrighted component of this work in other works.

Digital Object Identifier (DOI): 10.1109/ECCE.2015.7309750

IEEE Transactions on Power Electronics

Quad-active-bridge as cross-link for medium voltage modular inverters

Levy Ferreira Costa

Giampaolo Buticchi

Marco Liserre

Suggested Citation

L. F. Costa, G. Buticchi and M. Liserre, "Quad-active-bridge as cross-link for medium voltage modular inverters," IEEE Energy Conversion Congress and Exposition (ECCE), Montreal, 2015, pp. 645-652. 


\title{
Quad-Active-Bridge as Cross-Link for Medium Voltage Modular Inverters
}

\author{
Levy F. Costa, Giampaolo Buticchi, Marco Liserre \\ Chair of Power Electronics, Technical Faculty \\ Christian-Albrecht-University of Kiel \\ Kaiserstr 2, 24113, Kiel, Germany \\ Email: lfc@tf.uni-kiel.de
}

\begin{abstract}
The Smart Transformer, a Solid-State transformer with control functionalities, is a promising smart grid technology. One of the main challenge of this system lies in making available a Medium-Voltage dc link, which can be tackled with modular solution. Thus, several isolated dc-dc converter can be used as a basic modules. In this paper, a Quad-Active-Bridge (QAB) dcdc converter is used as modules for the entire dc-dc conversion stage of a ST. Besides the feature of high power density and soft-switching operation (also found in others converters), this converter offers an additional power path, named "MV crosslink" through the HF transformer in the Medium-Voltage (MV) side, increasing the power controllability among the MV cell without involving the $L V$ side. To ensure soft-switching for all operation range of the QAB converter, the triangular current mode modulation strategy, previously study for the dual-activebridge converter, is extended to the $\mathrm{QAB}$ converter in this work. In addition, it is considered to use in the MV side the $\mathrm{H}$-Bridge cell and the multilevel neutral-point-clamped cell, to reduce the required voltage rating of the semiconductors. The theoretical analysis is performed for both converters. The theoretical analysis is verified by simulation and experimental results.
\end{abstract}

\section{INTRODUCTION}

In recent years, the smart grid technologies have received more and more attention, as a feasible solution to manage in a efficient way the growth in load and the high penetration of distributed generation (DG). One of these technologies is the Smart Transformer, which is a Solid-State Transformer [1] - [2] with control and communication functionalities [3]. This power electronics based system uses a high frequency (HF) transformer, reducing volume and space, and it can also provide ancillary services to the grid, such as: power factor correction, active filtering, VAR compensator, electronics protection and disturbance rejection [1] - [2].

The three-stage ST is usually composed by a MediumVoltage (MV) ac-dc stage, a HF isolated dc-dc stage and a Low-Voltage (LV) dc-ac stage. The main challenge of this architecture is the dc-dc conversion stage, since it has strict requirements, such as: high rated power, high current capability in LV side, high voltage capability in HV side, high frequency isolation and high efficiency. To meet all requirements, two solutions have been widely investigated: the first one is to use standard converter with high voltage rating devices [4] - [6], while the second one is based on the modular concept, in which several modules are used to share the total voltage and power among them [7] - [13]. Although

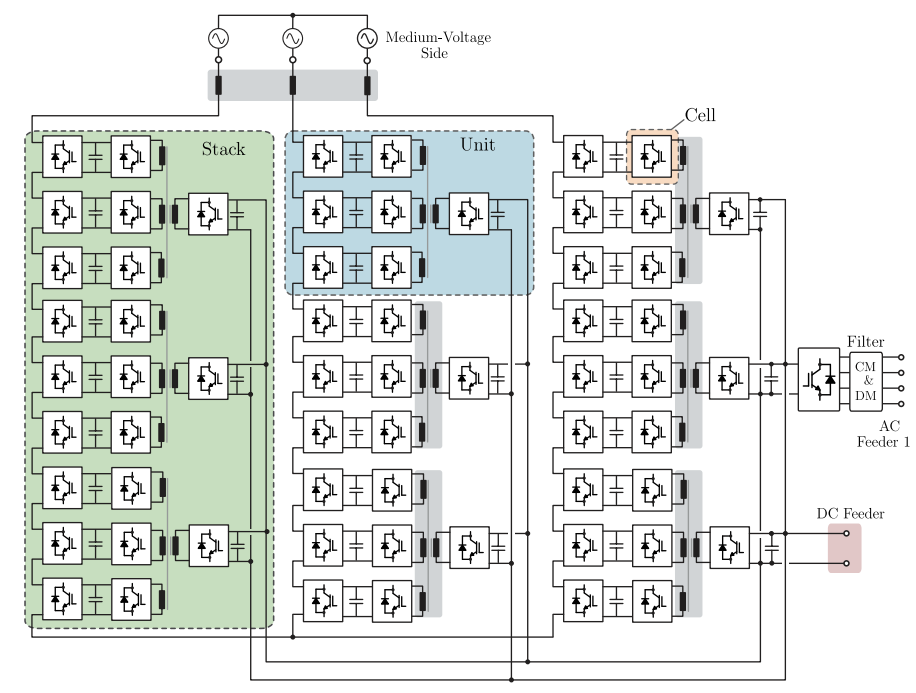

Fig. 1. architecture of the Smart Transformer employing the QAB converter as a solution for the dc-dc stage.

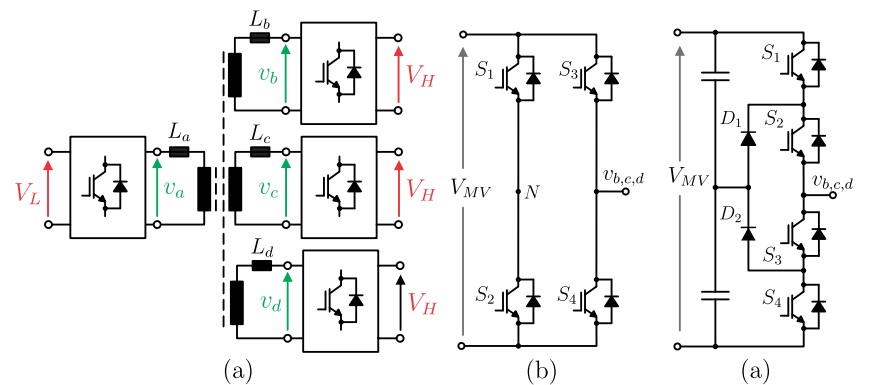

Fig. 2. Topology of the Quad-Active-Bridge converter: (b) Full-bridge converter and (c) NPC converter as a possible active bridge for the dc-dc converter.

the modular solution presents high number components, it has several advantages compared to the fist solution, such as: low $d v / d t$ (low EMI emission), possibility to use standard low voltage rating devices and also modularity, which allows to implement redundant strategy to increase the fault tolerance and reliability.

Several converters have been investigated to be used as module of the main core of the ST, but the Dual-Active-Bridge (DAB) and the Series-Resonant converter (SR) have received more attention, due its advantages of soft-switching, high efficiency and power density [8] - [13]. The Series-Resonant dc- 

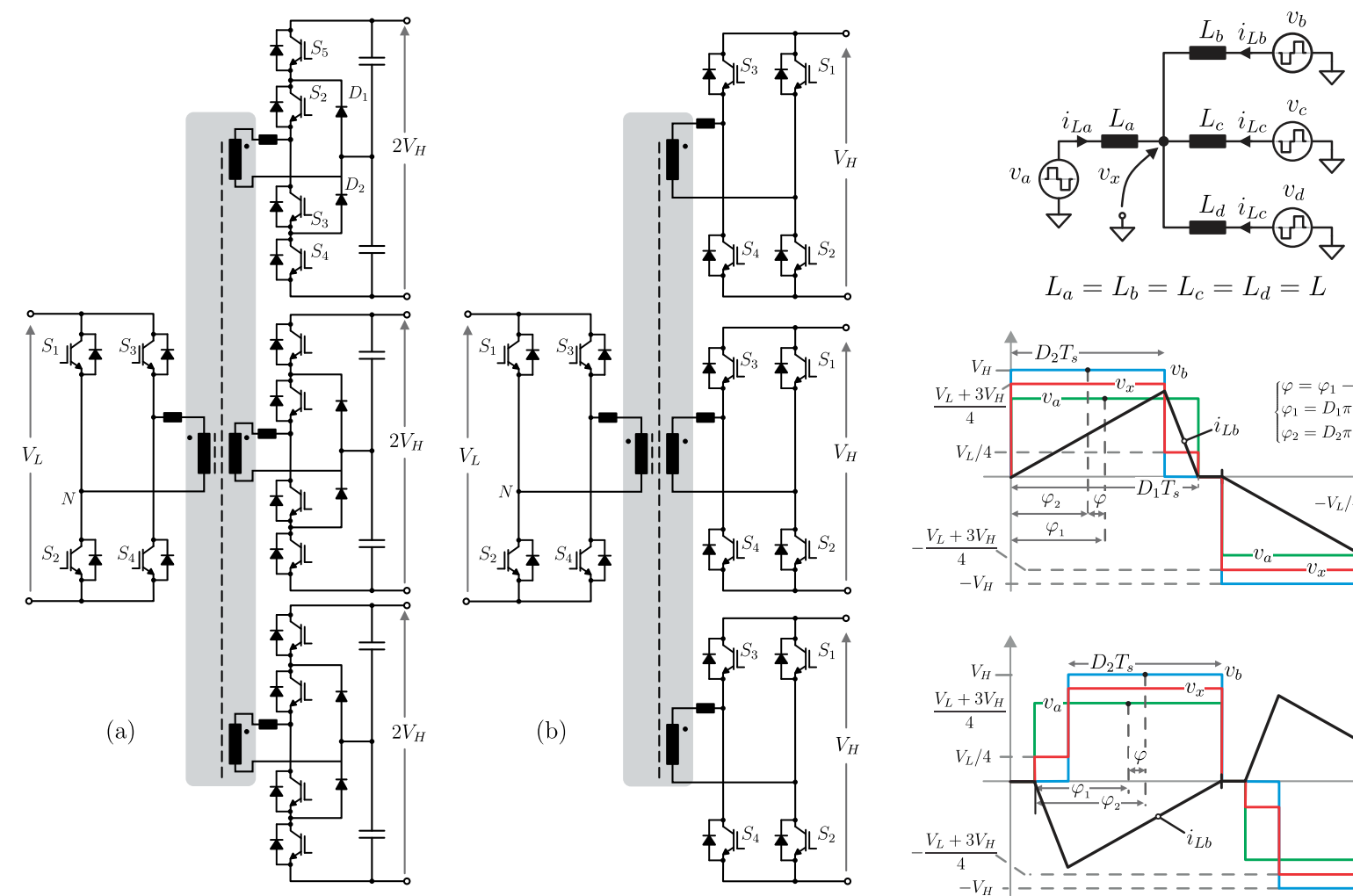

$$
L_{a}=L_{b}=L_{c}=L_{d}=L
$$
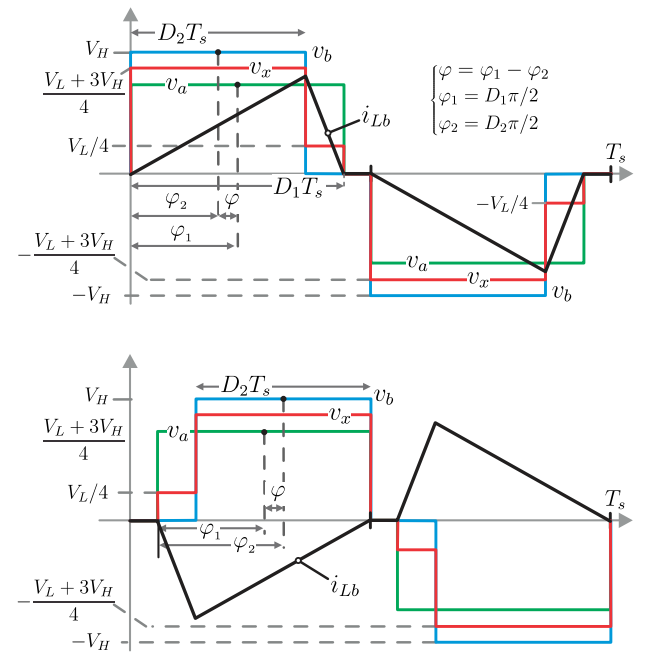

Fig. 3. Complete topology of the QAB dc-dc converter (a) using the NPC converter in the MV side and (b) using the HB converter in the MV side. (c) Equivalente circuit of the $\mathrm{QAB}$, used in the theoretical analysis of the converter. Main waveforms of the converter, when it is processing (d) positive power (from MV to LV) and (e) negative power flow (from LV to MV).

dc converter presents a well regulated output voltage for large range of load (when operating in discontinuous-conductionmode), avoiding the requirement of control loops. For that reason, it is also called dc-transformer [11] - [13]. On the other hand, when controllability of the output voltage or power is required, the $\mathrm{DAB}$ converter is more advantageous, since it works with active control of the transferred power [8] - [10]. In addition, this converter can also control the power flow among the MV modules of the ST, however the LV bridge and the LVDC link are involved in the power conversion path, compromising the overall efficiency.

To reduce the number of power conversion during the power exchange of the MV modules, a converter that provides a direct power path among the MV modules can be used instead the DAB converter. The Quad-Active-Bridge (QAB) dc-dc converter, previously reported in [14] - [16], has all the described characteristics. This converter is composed by four actives bridges connected to the same four winding $\mathrm{HF}$ transformer, as depicted in Fig. 2 (a). For ST application, one bridge of the $\mathrm{QAB}$ converter should be connected to the $\mathrm{LV}$ side and the others three bridges should be connected to the MV side, in order to share the Medium-Voltage among them. In this configuration, the $\mathrm{QAB} \mathrm{dc}-\mathrm{dc}$ converter has the same advantages of the DAB, however with an additional feature of power transfer capability among the MV cells in a high efficiency way. As can be seen in Fig. 2, the QAB has a magnetic link that allow directly exchange of power among the
MV cells without including the LV cell In others words, the QAB offers an additional power path to the MV cells, named "cross-link", which can increase the redundancy scheme and, consequently, the reliability of the system.

Similarly to the DAB converter, the QAB converter can also work with phase-shift modulation, where the power changed among two bridges is controlled by the shifted angle of one bridge in respect to the other. However, this modulation strategy might have some limitation on the soft-switching operation, when the input or output voltage varies. To overcome this problem, duty-cycle variation can be used to ensure softswitching operation of the converter, as reported in [17].

In this context, this paper investigate the application of the QAB converter using the triangular current modulation strategy to a Smart Transformer, focusing only on the power converter performance. Fig. 1 shows the the block diagram of a ST employing the QAB converter as dc-dc solution and a Cascaded H-Bridge (CHB) for the MV ac-dc conversion stage. In this work, a power unit (highlighted in Fig. 1) can be defined as a replaceable part of the system. For this architecture, an unit is composed by an entire $\mathrm{QAB}$ converter and some modules of the $\mathrm{CHB}$ converter, but only those associated to the QAB converter of the unit, as can be seen in Fig. 1. The power cell is the smallest power block of the unit and it is also highlighted in Fig. 1. In architecture, it is chosen to use three units per phase, to have a good trade-off between number of components and effort among the same. 
TABLE I

SPECIFICATION OF A SMART TRANSFORMER APPLIED TO DISTRIBUTION SYSTEM

\begin{tabular}{c|c|c|c}
\hline \hline Specification & Smart Transformer & Specification & QAB \\
\hline Rated Power & $1 \mathrm{MVA}$ & Rated Power & $111 \mathrm{~kW}$ \\
\hline Input Voltage & $700 \mathrm{~V}$ & Input voltage (LV) & $700 \mathrm{~V}$ \\
\hline Output Voltage & $10 \mathrm{kV}$ & Output voltage (MV) & $1.13 \mathrm{kV}$ \\
\hline LVDC link & $700 \mathrm{~V}$ & Switching frequency & $20 \mathrm{kHz}$ \\
\hline MVDC link & $10.2 \mathrm{kV}$ & IGBT & $1200 \mathrm{~V}$ \\
\hline
\end{tabular}

Table I shows the specification of a typical ST for distribution system application and also the specification of a single QAB converter of the ST, considering the configuration shown in Fig. 1. As it can be observed, the voltage of the MV bridges of the QAB converter is still high $(1.13 \mathrm{kV})$. Using a standard H-bridge cell in the MV side, $1.7 \mathrm{kV}$ IGBT are required. However, multilevel cell can also be employed in the MV side, in order to reduce the voltage of the semiconductors and to enable to use of $1.2 \mathrm{kV}$, which presents lower forward drop voltage and lower conduction losses. In this work, the NPC cell is also considered to be used in the MV cell of the QAB converter. Fig. 3 (a) and (b) shows the complete topology of the QAB dc-dc converter, employing NPC cell and HB cell in the medium voltage, respectively. Both cells are considered for study in this paper.

The main contribution of this paper in the field of QAB, is to evaluate the operation of the $\mathrm{QAB}$ converter using the triangular current modulation strategy for smart transformer application, where all the main equations for the design are derived. As an additional contribution, the operation of the QAB converter using a NPC cell is also investigate in this paper. The theoretical analysis is carried out considering the balancing condition (all MV bridges processing the same amount of power) and also unbalancing condition (all MV bridges processing the different amount of power) of the QAB converter. The operation principle of the QAB converter using the triangular current modulation strategy is presented in section II for balance condition, where the modulation strategy is described and the equations for the power transferred and current effort are described. The unbalance condition is discussed in section III. Finally, the simulation and experimental results are presented and discussed in section IV and the conclusion is presented in section $\mathrm{V}$.

\section{OPERATION PRINCIPLE}

The topology of the QAB is shown in Fig. 3 (a) and (b), considering different cells on the MV side. Regardless the cell, an equivalent circuit based on Y-model can be used to analyze the converter. In this equivalent circuit, the bridges are replaced by rectangular voltage source $\left(v_{a}, v_{b}, v_{c}\right.$ and $\left.v_{d}\right)$ (see Fig. (2)) and the voltage of the central point $v_{x}$ is given by (1). The current slope of each bridge can be defined by (2), where $\mathrm{k}=\{\mathrm{a}, \mathrm{b}, \mathrm{c}, \mathrm{d}\}$. As defined in Fig. 2, $v_{a}$ is the $\mathrm{ac}$ voltage synthesized by the LV bridge, while the voltage $v_{b}, v_{c}$ and $v_{d}$ are the voltage of the MV cells. The power processed by the bridges named $a, b, c$ and $d$ are $P_{1}, P_{2}, P_{3}$ and $P_{4}$, respectively, and $P_{1}=P_{2}+P_{3}+P_{4}$. As the $\mathrm{HB}$ converter is able to synthesize twice the total dc voltage $\left(+V_{M V}, 0\right.$ and $\left.-V_{M V}\right)$, while the NPC converter can synthesize only the total de voltage $\left(+V_{M V} / 2,0\right.$ and $\left.-V_{M V} / 2\right)$, the modulation analysis is carried out considering the HB converter. In this analysis, it is also considered that the all inductors have the same inductance value $\left(L_{1}=L_{2}=L_{3}=L_{4}=L\right)$ and also that the power is transferred from the MV side to the LV side.

$$
\begin{gathered}
v_{x}=\frac{v_{a}+v_{b}+v_{c}+v_{d}}{4} \\
\frac{d i_{L k}}{d t}=\frac{\left(v_{k}-v_{x}\right)}{L}
\end{gathered}
$$

\section{A. Triangular Current Mode Modulation Strategy}

To modulate the converter, the Triangular Current Modulation Strategy (TCMS) presents an attractive solution [18]. By properly selecting the transformer turns ratio, the MV side switches can be operated in ZCS mode in both power directions while actively controlling the transferred power by adjusting the LV side duty cycle [17]. This strategy has been intensively investigated for the DAB converter [17] - [20], and in this paper, it is extended to the $\mathrm{QAB}$ converter.

Initially, it is considered that the QAB operates balanced, i.e. each MV bridge processes the same amount of power (P2 $=\mathrm{P} 3=\mathrm{P} 4)$. In this case, low voltage bridge operates with duty-cycle $d_{1}$ and all the three MV bridges operate with the same duty-cycle $d_{2}$.

The basic principle of this modulation strategy is to impose a triangular current on the inductors, as shown in Fig. 3 (d) and (e). To do that, the voltages $v_{a}$ and $v_{b}$ should have the waveforms shown in Using this modulation strategy, the voltage $v_{x}$ is given by (3) for this condition. The current $i_{L b}$, the voltages $v_{a}, v_{b}$ and $v_{x}$ are shown in Fig. $3(\mathrm{~d})$, for positive power flow, and (e) for negative power flow. As it can be observed, the current through the inductor starts from zero and reaches its maximum value $\Delta i_{L b}$ during the period of time between 0 and $D_{1} T_{s}$, where $T_{s}$ is the switching period. The current variation during this period is described by (4). As the current starts from zero, the switches $S_{2}$ and $S_{3}$ of the LV side and also the switches $S_{5}$ and $S_{6}$ of the MV side turns-on at Zero-Current-Switching (ZCS). In this moment ( $t=D_{1} T_{s}$ ), the Zero-Voltage-Switching will occur, but only if energy stored in the transformer inductance is enough to completely discharge the total equivalent output capacitance of the switch, as seen by the circuit, before it is switched on. Energy stored in the inductor depends on squared value of the peak current $\left(\Delta i_{L b}\right)$ at the moment transition occurs, while the total equivalent output capacitance of the switch includes switchs built-in output capacitance, additional snubber and parasitic capacitances. Thus, the capacitors snubber should be designed to achieve the ZVS commutation. 


$$
v_{x}=\left\{\begin{array}{cc}
\frac{n V_{L}+3 V_{H}}{4}, \quad 0<t<d_{2} T_{s} \\
\frac{n V_{L}}{4}, \quad d_{2} T_{s}<t<d_{1} T_{s} \\
0, \quad d_{1} T_{s}<t<T_{s} / 2 \\
\Delta i_{L b_{\left(0<t<D_{2} T_{s}\right)}}=\frac{D_{2}\left(V_{H}-n V_{L}\right)}{4 L f}
\end{array}\right.
$$

Likewise, during the period between $D_{1} T_{s}$ and $D_{2} T_{s}$, the current decrease from $\Delta i_{L b}$ until reaches zero again. This variation is described by (5). In the instant $D_{2} T_{s}$, the current is zero and the switch $S_{1}$ turned-off (at ZCS) and the MV bridge imposes $v_{a}=0$. It is important to note that the period in zero state should be long enough to evacuate all charge accumulated at the IGBT during the conduction state. Thus, this commutation can be critical for rated load operation, where the stored charger is high and the zero time period is small [17]. To achieve ZCS operation regardless the load and input or output voltages levels, the condition $(4)=(5)$ must be satisfied. As a results, the relation between the duty-cycle $D_{1}$ and $D_{2}$ is found and presented in (6).

$$
\begin{gathered}
\Delta i_{L b_{\left(D_{2} T_{s}<t<T_{s} / 2\right)}}=\frac{n V_{L}\left(D_{1}-D_{2}\right)}{4 L f_{s}} \\
D_{2}=\frac{V_{L} \cdot n}{V_{H}} D_{1}
\end{gathered}
$$

\section{B. Power Transferred}

The transfered power can be calculated on the dc side of the bridges by: $P_{k a v g}=I_{k} V_{k}$, where $k$ denotes the cell. The average current in the cell $b\left(I_{b}\right)$ is calculated in (7). Thus, the power processed by the cell $b$ (MV cell) and $a$ (LV cell) are shown in (8).

$$
\begin{gathered}
I_{b}=\frac{2}{T_{s}} \int_{0}^{D_{2} T_{s}} i_{b}(d) d t=\frac{D_{2}^{2}\left(V_{H}-n V_{L}\right)}{4 L f_{s}} \\
P_{2}=\frac{D_{1}^{2}\left(V_{L} n\right)\left(V_{H}-V_{L} n\right)}{4 L f_{s}} \\
P_{1}=\frac{3 D_{1}^{2}\left(V_{L} n\right)\left(V_{H}-V_{L} n\right)}{4 L f_{s}}
\end{gathered}
$$

\section{Current Efforts}

From Fig. 3 (d), it is possible to calculate the current effort on the primary (LV side) and secondary (LV side) of the transformer. The rms current on the primary and secondary sides in function of the duty-duty $D_{1}$ is shown in (9) and (10).

$$
\begin{aligned}
i_{\mathrm{sec}, r m s} & =\frac{n V_{L}\left(V_{H}-n V_{L}\right)}{12 L f_{s} V_{H}} \sqrt{6 d_{1}{ }^{3}} \\
i_{\text {prim }, r m s} & =\frac{n^{2} V_{L}\left(V_{H}-n V_{L}\right)}{4 L f_{s} V_{H}} \sqrt{6 d_{1}{ }^{3}}
\end{aligned}
$$
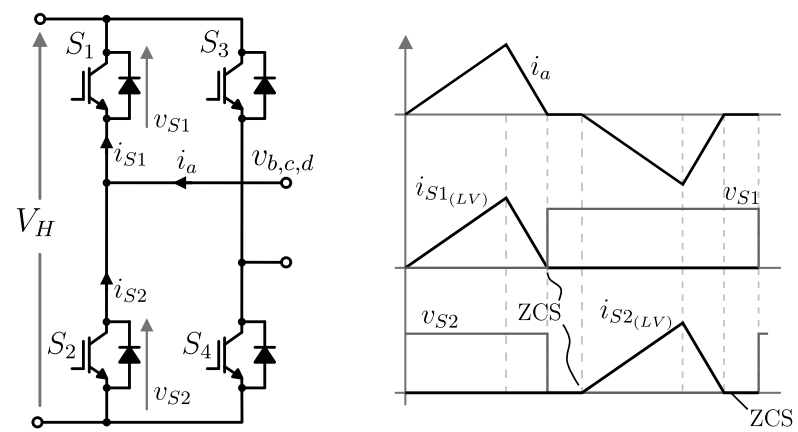

(a) LV cell
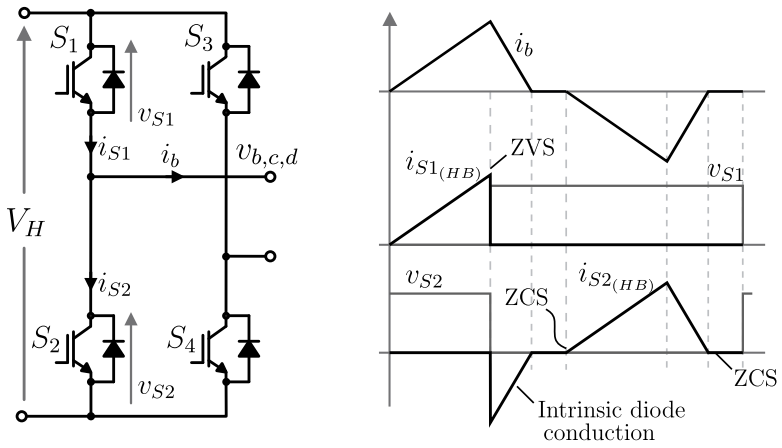

(b) MV cell based on HB topology
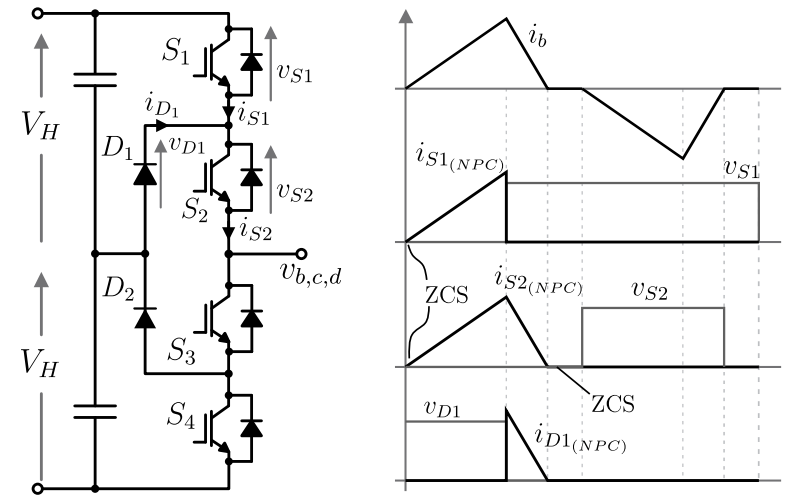

(c) MV cell based on NPC topology

Fig. 4. (a) Topology of the LV cell with the current and voltage waveforms of the switches, (b) topology of the MV cell based on NPC converter with the current and voltage waveforms of the switches.

The current waveform in each switch of the QAB converter is depicted in Fig. 4. Fig. (4) shows the current for the LV side bridge, while the Figs. (4) (b) and (c) shows the current for the MV side bridge, when it is implemented with $\mathrm{HB}$ and NPC, respectively.

As can be seen, the LV side switches commutate at ZCS during the turn-on and turn-off, as well the switches of the MV cell. For the switch $S_{2}$ of the MV cell and LV cell (see Fig. 4), the commutation are complete free of losses, since the current remains in zero for a while before the voltage starts to increase.

The current efforts in the LV bridge can be calculated from Fig. 4 (a) and efforts (rms and mean) for each semiconductors 
of the MV cell are presented from equation (11) to (12).

$$
\begin{aligned}
& i_{s_{1(L V)}, r m s}=\frac{n^{2} V_{L}\left(V_{H}-n V_{L}\right)}{4 L f_{s} V_{H}} \sqrt{3 d_{1}{ }^{3}} \\
& i_{s_{1(L V)}, a g v}=\frac{3 d_{1}{ }^{2}\left(n^{2} V_{L}\right)\left(V_{H}-n V_{L}\right)}{8 L f_{s} V_{H}}
\end{aligned}
$$

Similarly, the current efforts for the MV side, when the NPC cell is amployd, is obtained from Fig. 4 (c) and the efforts (rms and mean) for each semiconductors of this cell are presented from equation (13) to (18).

$$
\begin{gathered}
i_{s_{1}, r m s}=\frac{n V_{L}\left(V_{H}-n V_{L}\right)}{12 L f_{s} V_{H}} \sqrt{\frac{3 d_{1}^{2} n V_{L}}{V_{H}}} \\
i_{s_{1}, a g v}=\frac{d_{1}^{2}\left(n V_{L}\right)^{2}\left(V_{H}-n V_{L}\right)}{8 L f_{s} V_{H}^{2}} \\
i_{s_{2}, r m s}=\frac{n V_{L}\left(V_{H}-n V_{L}\right)}{12 L f_{s} V_{H}} \sqrt{3 d_{1}^{3}} \\
i_{s_{2}, a g v}=\frac{d_{1}^{2}\left(n V_{L}\right)\left(V_{H}-n V_{L}\right)}{8 L f_{s} V_{H}} \\
i_{D_{1}, r m s}=\frac{n V_{L}\left(V_{H}-n V_{L}\right)}{12 L f_{s} V_{H}} \sqrt{\frac{3 d_{1}^{3}\left(V_{H}-n V_{L}\right)}{V_{H}}} \\
i_{D_{1}, a g v}=\frac{d_{1}^{2}\left(n V_{L}\right)\left(V_{H}-n V_{L}\right)^{2}}{8 L f_{s} V_{H}^{2}}
\end{gathered}
$$

For the MV side using the HB cell, the current efforts on the semiconductors are very similar to the NPC case. As can be observed in Fig. 4 (b) and (c), the waveform for the current $i_{S 1_{H B}}$ of the $\mathrm{HB}$ cell is similar to the current $i_{S 1_{N P C}}$ of the NPC cell. Thus, the current effort is defined by the same equation and already presented in (13) and (14). For the switch $S_{2}$ of the $\mathrm{HB}\left(i_{S 2_{H B}}\right)$, part of the current flows through the channel (positive part) and the other part flows through the diode (negative part). Thus, the effort can be calculate separately in this two element of the devices. The current the flows through the channel of the IGBT is similar to the current $i_{S 2_{(N P C)}}$, then it can be calculated using also equations (15) and (16). Likewise, the current flowing through the intrinsic diode of the device $\left(S_{s}\right.$ of the HB cell) is similar to current $i_{D 1_{(N P C)}}$ and it can be calculated by (17) and (18). It is important to note that the equation to calculate the effort on the NPC cell and HB cell are very similar, but the parameters (mainly $n, L$ ) are different for the same output power. The current peak in the NPC cell is twice the current peak on the HB cell.

From these equation, the rms current on the primary side and secondary side in function of the output power are plotted in Fig. 5 (a), while the current efforts on the semiconductors are depicted in Fig. 5 (b).
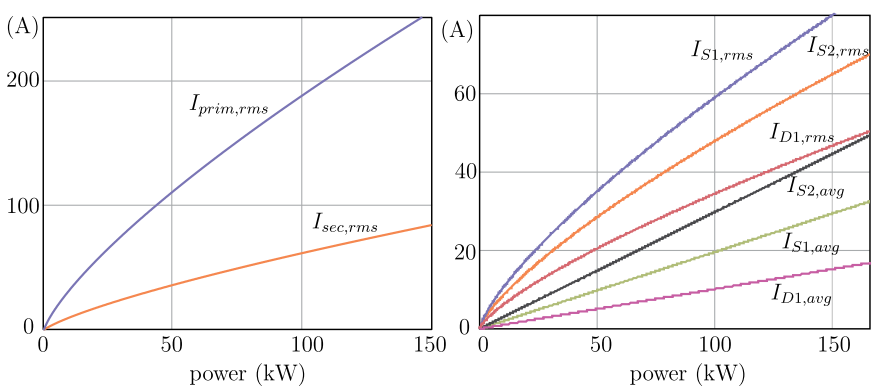

Fig. 5. Current efforts in function of the power: (a) rms current on the primary side and secondary side of the QAB converter, (b) rms and mean current on the switches of the MV side of the QAB converter.

\section{UnBALANCE CONDITION}

For unbalance condition $\left(P_{2} \neq P_{3} \neq P_{4}\right)$, the duty cycle of the MV bridges are not equal anymore. The duty-cycle of each MV cell will depend on the power processed by them. For this analysis, it is considered that only the power of the cell $b$ is different, i.e. $P_{2} \neq P_{3}=P_{4}$. Considering this condition, the voltages $v_{a}, v_{b}$ and $v_{x}$, as well as the current $i_{b}$ and $i_{c}$ are depicted in Fig. 6. As it can be observed, the current starts from a value different o zero, increase until reaches the maximum value, and them the current starts to decrease again until to reaches the same negative initial value. During the commutation, the current is not zero anymore, hence the ZCS feature is lost. Nevertheless, the current value during the commutation is very low, which indicates a very low switching losses. Actually, the switching losses will be proportional to the unbalanced load.

As the cell $b$ is processing different amount of power compared to the cells $c$ and $d$, then its duty-cycle is also slightly different. The duty cycle of the cell $b$ is $D_{2}$, while the duty cycle of the cells $c$ and $d$ is defined as $D_{3}$. D3. The slight difference of the duty-cycle cell $b$ regarding the dutycycle of cell $c$ and $d$ is defined as $\Delta D_{2}=D_{2}-D_{3}$.

As can be observed in Fig. 6, in the instant $t=D_{2} T_{s}$ the voltage $v_{b}$ goes to zero, but the voltage $v_{c}$ and $v_{d}$ remains equal to $V_{H}$ until the instant $t=D_{3} T_{s}$. Thus, the voltage $v_{x}$ will have one more level, as can be seen in that figure. This additional level makes the current $i_{b}$ decreases and the current $i_{c}$ increases. Thus, the currents $i_{b}$ and $i_{c}$ has different behavior during this period of time between $D_{2} T_{s}$ and $D_{3} T_{s}$ (or called $\Delta D_{2}$ ). Afterwards, both current decreases with the same slope, but they achieve different values, because they start from different points. As a conclusion, the variable $\Delta D_{2}$ has direct impact on the dc value of the currents, and consequently the additional power delivered by the MV cell. For that reason, this new variable can be used to control the power exchange among the MV cells. The Figs. 7 (a) and (b) show the current waveforms of the cells $b$ and $c$, respectively, for balancing and unbalancing condition. As it can be seen, the output current of the ports are affected by the unbalancing condition only during half switching period. Besides that, the additional charge delivered or removed from each port is highlighted in 


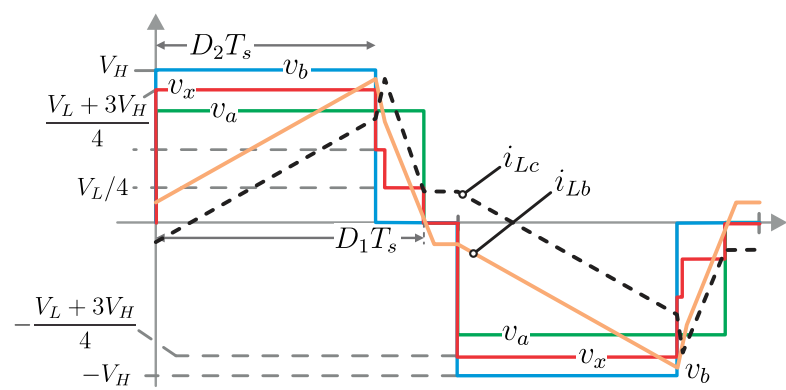

Fig. 6. Main waveforms of the converter for unbalance condition and also for positive power flow.
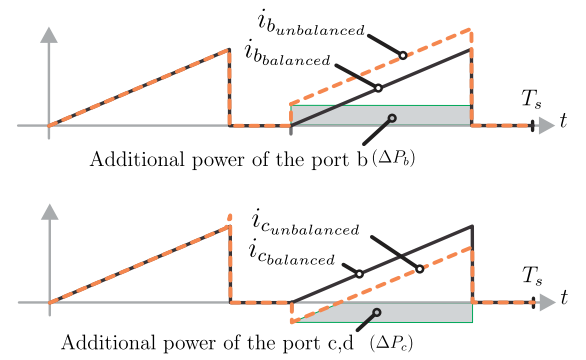

Fig. 7. current waveform on the dc side of the cell $b$ and $c$, highlighting the additional charger delivered or removed from these cells.

the figure.

$$
\begin{gathered}
\Delta P_{2}=V_{H} \frac{\Delta Q_{2}}{T_{s}} \rightarrow \Delta Q_{2}=\int_{T_{s} / 2}^{T_{s}} i b(t) d t \\
P_{2}=\frac{P_{1}}{3}+\Delta P_{2}
\end{gathered}
$$

The power processed by the cell $b$ is composed by the total balanced power (given by $P_{1} / 3$ ) and an additional power portion $\Delta P_{2}$, as presented in (20). The variable $\Delta P_{2}$ is responsible for the unbalancing power and for power balanced case, we have $\Delta P_{2}=0$. The additional power $\Delta P_{2}$ is calculated using the additional charge shown in Fig. 7 (b) as presented in (19). The additional charge is also shown in equation (19). Therefore, solving (19), it is found the equation of $\Delta P_{2}$ in function of $\Delta D_{2}$, as shown in (21). The same procedure can be done to the power transferred by the cell $c$ and as a result, the power processed by the cell $c$ is given by (21). As it can be observed in (12) and (13), the equations of the additional power are in function of $\Delta D_{2}$, then this variable can be use to control the additional power transferred to the MV bridges.

$$
\begin{aligned}
\Delta P_{2} & =\frac{3 V_{H} \Delta D_{2}\left(D_{2}-\Delta D_{2}\right)}{2 L f_{s}} \\
\Delta P_{3} & =\frac{3 V_{H} \Delta D_{2}\left(\Delta D_{2}-D_{2}\right)}{4 L f_{s}}
\end{aligned}
$$

TABLE II SiMULATION PARAMETERS

\begin{tabular}{c|c}
\hline \hline Specification & Smart Transformer \\
\hline Power & $166 \mathrm{~kW}$ \\
\hline Input Voltage $(\mathrm{MV})$ & $1700 \mathrm{~V}$ \\
\hline Output Voltage $(\mathrm{LV})$ & $700 \mathrm{~V}$ \\
\hline Switching frequency & $20 \mathrm{kHz}$ \\
\hline Inductance & $4.6 \mu \mathrm{H}$ \\
\hline Transformer turn ratio & $\mathrm{n}=0.8$ \\
\hline \hline
\end{tabular}

\section{Simulation And ExPERimental Results}

To evaluate the performance of the Quad-Active-Bridge converter using the described modulation strategy and also to verify the theoretical analysis developed in this paper, simulation and experimental results were obtained and they will be discussed in this section.

\section{A. Simulation Results}

The simulations was carried out with MATLAB/Simulink and the PLECS toolbox and it is based on the parameters specified in Table II. In addition, leakage inductance of 10 $\mu \mathrm{H}$ and transformer ratio of $n=2.63$ were used.

Fig. 8 (a) to (c) shows the main waveforms in steady-state of the QAB under balancing condition, while Fig. 8 (d) to (e) shows the same waveforms for unbalancing condition. In Fig. 8 (a), it is depicted the voltages $v_{a}, v_{b}$ and $v_{x}$ ans the currents $i_{a}, i_{b}, i_{c}$ and $i_{d}$, similarly to the theoretical waveforms illustrated in Fig. 3 (d). The current and voltage waveforms on the switches of the MV bridge and LV bridge are presented in the Fig. 8 (b) and (c), respectively. In this figures, the softswitching operation is clearly observed and waveforms are similar to those theoretical ones shown in Fig. 4.

For unbalanced condition (Fig. 8 (d) to (e)), it is considered that the total power processed by the $\mathrm{QAB}$ converter is the same of the previous condition, i.e. $166 \mathrm{~kW}$, however it is also assumed the cell $b$ is processing $25 \mathrm{~kW}$, while the cells $c$ and $d$ are processing around $43 \mathrm{~kW}$ each. The unbalancing effect can be clearly observed on the waveform of the voltage $v_{x}$ (Fig. 8 (d) on top), in which more levels are presented on this waveforms, and also on the waveforms of the currents $i_{b}$ and $i_{c}$. The waveforms obtained by simulation are identical to the theoretical one shown in Fig. 6. The current and voltage waveforms on the switches of the MV bridge and LV bridge are presented in the Fig. 8 (e) and (f). In this condition, the soft-switching feature is lost. The hard-switching commutation is even more clear to be observed in the Fig. 8 (f), where the current starts and finish in a value different of zero. Even with a considerably difference of power processed by the cell $b$ and $c$, the current level on the switches during the commutation is considerably low, implying also in low switching losses.

\section{B. Experimental Results}

To evaluate the performance of the Quad-Active-Bridge dc-dc converter experimentally, a downscale prototype was 



(a) Main waveform in steady-state for balance condition
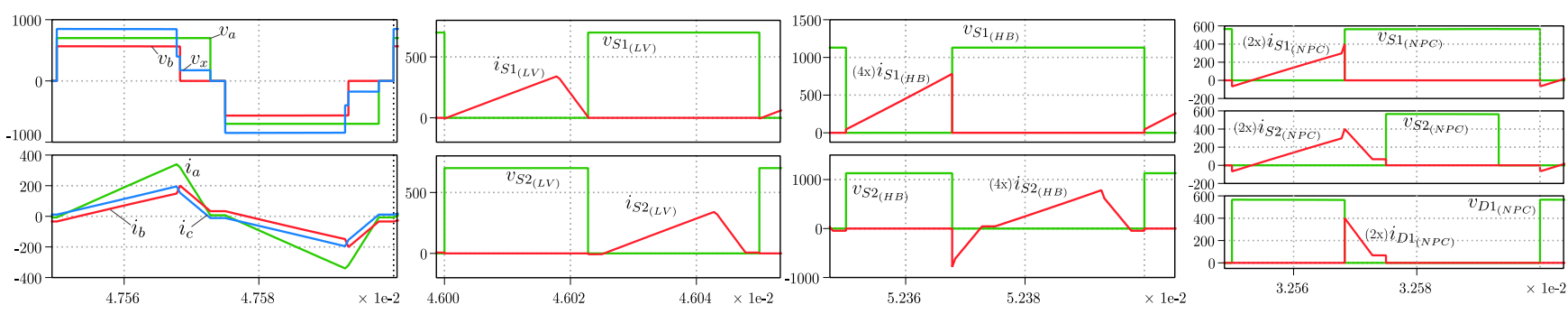

(b) Main waveform in steady-state for unbalance condition

Fig. 8. Main steady-state waveforms of the QAB dc-dc converter for balanced (from (a) to (c)) and unbalance (from (d) to (e))

TABLE III

EXPERIMENTAL SETUP PARAMETERS

\begin{tabular}{c|c}
\hline \hline Specification & Smart Transformer \\
\hline Power & $20 \mathrm{~kW}$ \\
\hline Input Voltage (MV) & $800 \mathrm{~V}$ \\
\hline Output Voltage (LV) & $800 \mathrm{~V}$ \\
\hline Switching frequency & $20 \mathrm{kHz}$ \\
\hline Inductance & $50 \mu \mathrm{H}$ \\
\hline Transformer turn ratio & $\mathrm{n}=1$ \\
\hline
\end{tabular}

designed, built and experimental results were obtained. The prototype is based on the structure shown in 3 (b), i.e. with $\mathrm{HB}$ in the MV side, and it was tested with the triangular current mode modulation strategy for validation purposes. The electrical parameters of the prototype are shown in Table III. The tests were carried out with resistive load. The results were obtained for a input voltage of $250 \mathrm{~V}$ and output voltage of $200 \mathrm{~V}$. The results are depicted in Fig. 9 and 10, for balance and unbalance conditions, respectively.

In Fig. 9, the voltage on LV side (on the cell $a$ ) and MV side (on the cells $b$ and $c$ ) as well as the current on the LV side $i_{a}$ are depicted. As can be seen, the voltages $v_{b}$ and $v_{c}$ have the same value, indicating balanced condition. In this figure, a perfect triangular current shape is observed and it is very similar to the simulated and theoretical waveforms. Besides that, the soft-switching is clear in this figure.

Finally, the experimental waveform for unbalanced condition operation is shown in Fig. 10, where different value for the voltage $v_{b}$ and $v_{c}$. Besides that, in this condition it is very clear that the converter does not operate at soft-switching anymore,

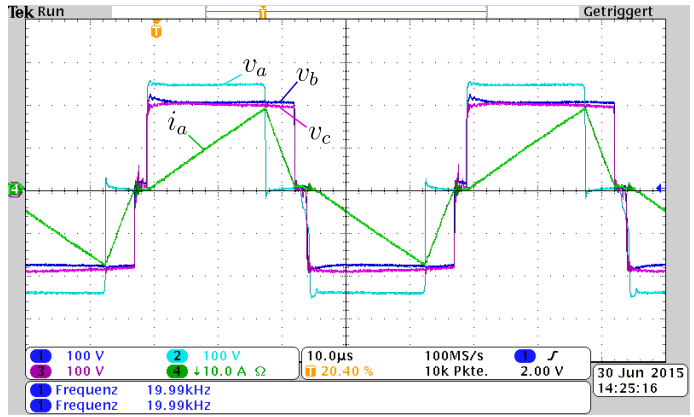

Fig. 9. Experimental results of the QAB converter based on the HB topology in the MV side, using the TMSM for balanced condition.

as already discussed.

\section{CONClusion}

In this paper the modulation strategy and operation principle of the medium voltage Quad-Active-Bridge as a solution for the dc-dc conversion stage of smart transformers was presented. The topology presents several advantages in this application, such as soft-switching operation and high power density. In addition, the topology offers an additional power path in the medium voltage side, enabling the power exchange among the MV cells in a efficient way, and increases the redundancy, consequently, the reliability of the system.

The focus of this paper is to study the modulation and operation of the MV QAB dc-dc converter. The triangular current modulation strategy, previously study for the DAB, has its application extended to the QAB converter in this work, where the basic equations of the modulation for this converter are derived. 


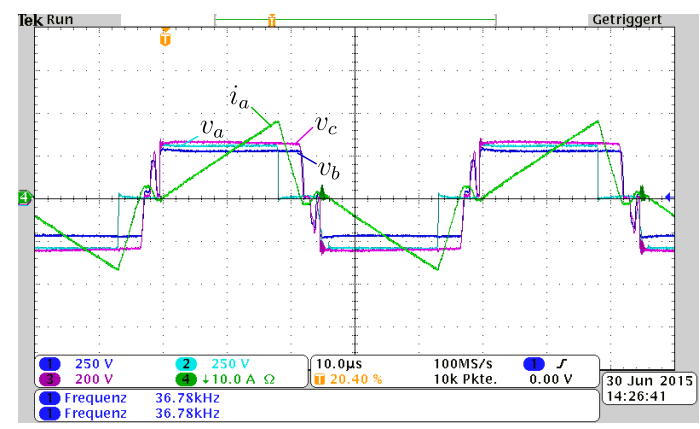

Fig. 10. Experimental results of the QAB converter based on the HB topology in the MV side, using the TMSM for unbalanced condition.

Moreover, the converter operation at unbalance power is also investigate and a control scheme for balance the voltage in the MV cell is presented. Simulation and experimental results are provided in this paper to attest the theoretical analysis developed herein. The results are in accordance with the theoretical waveforms. As a overall overview, the Quad-ActiveBridge converter has presented high performance with NPC in the MV side, which make it suitable for SST application.

\section{REFERENCES}

[1] X. She, R. Burgos, G. Wang, F. Wang, and A. Huang, "Review of solid state transformer in the distribution system: From components to field application", IEEE Energy Conversion Congress and Exposition (ECCE), Sept 2012, pp. 4077-4084.

[2] J. W. Kolar, G. Ortiz, "Solid-State-Transformers: Key Components of Future Traction and Smart Grid Systems", Proceedings of the International Power Electronics Conference - ECCE Asia (IPEC 2014), Hiroshima, Japan, May 18-21, 2014.

[3] De Carne, G.; Liserre, M.; Christakou, K.; Paolone, M., "Integrated voltage control and line congestion management in Active Distribution Networks by means of smart transformers", 23rd International Symposium on Industrial Electronics (ISIE), pp.2613-2619, June 2014

[4] N. Soltau, R. U. Lenke, R. W. De Doncker, "High-Power DC-DC Converter", Technical Report, September 2013

[5] S. Madhusoodhanan, A. Tripathi, D. Patel, K. Mainali, A.Kadavelugu, S. Hazra, S Bhattacharya, K. Hatua, "Solid State Transformer and MV Grid Tie Applications Enabled by $15 \mathrm{kV} \mathrm{SiC} \mathrm{IGBTs}$ and $10 \mathrm{kV} \mathrm{SiC}$ MOSFETs Based Multilevel Converters", IEEE Transactions on Industry Applications, (Early access).

[6] D. Rothmund, G. Ortiz, T. Guillod, J. W. Kolar, "10kV SiC-Based Isolated DC-DC Converter for Medium-Voltage-Connected SSTs", Proceedings of the 30th Applied Power Electronics Conference and Exposition (APEC 2015), Charlotte, NC, USA, March 15-19, 2015.

[7] J. Huber, J. W. Kolar, Volume/Weight/Cost Comparison of 1 MVA $10 \mathrm{kV} / 400$ V Solid-State against a Conventional Low-Frequency Distribution Transformer, Proceedings of the IEEE Energy Conversion Congress and Exposition (ECCE USA 2014), Pittsburgh, PA, USA, September 1418, 2014.

[8] Xu She; S. Lukic; A.Q. Huang, S. Bhattacharya, M. Baran, "Performance evaluation of solid state transformer based microgrid in FREEDM systems", Applied Power Electronics Conference and Exposition (APEC), 2011 Twenty-Sixth Annual IEEE, pp.182,188, 6-11 March 2011

[9] Haifeng Fan; Hui Li, "High-Frequency Transformer Isolated Bidirectional DCDC Converter Modules With High Efficiency Over Wide Load Range for $20 \mathrm{kVA}$ Solid-State Transformer", IEEE Transactions on Power Electronics, vol.26, no.12, pp.3599,3608, Dec. 2011

[10] Biao Zhao; Qiang Song; Wenhua Liu, "A Practical Solution of HighFrequency-Link Bidirectional Solid-State Transformer Based on Advanced Components in Hybrid Microgrid", IEEE Transactions on Industrial Electronics, vol.62, no.7, pp.4587,4597, July 2015
[11] Dujic, D.; Kieferndorf, F.; Canales, Francisco; Drofenik, U., "Power electronic traction transformer technology", 7th International Power Electronics and Motion Control Conference (IPEMC), vol.1, pp.636,642, 2-5 June 2012

[12] Chuanhong Zhao; S. Lewdeni-Schmid, J.K. Steinke, M. Weiss, T. Chaudhuri, M. Pellerin, J. Duron, P. Stefanutti, "Design, implementation and performance of a modular power electronic transformer (PET) for railway application", Proceedings of the 14th European Conference on Power Electronics and Applications (EPE 2011),, pp.1,10, Aug. 30 2011Sept. 12011

[13] D. Rothmund, J. Huber, J. W. Kolar, "Operating Behavior and Design of the Half-Cycle Discontinuous-Conduction-Mode Series-ResonantConverter with Small DC Link Capacitors", Proceedings of the 14th IEEE Workshop on Control and Modeling for Power Electronics (COMPEL 2013), Salt Lake City, USA, June 23-26, 2013.

[14] Tao, H.; Kotsopoulos, A.; Duarte, J.L.; Hendrix, M.A.M., "Family of multiport bidirectional DC-DC converters", IEE Proceedings Electric Power Applications - , vol.153, no.3, pp.451,458, 1 May 2006

[15] Falcones, S.; Ayyanar, R.; Xiaolin Mao, "A DC-DC Multiport-ConverterBased Solid-State Transformer Integrating Distributed Generation and Storage", IEEE Trans. on Power Electr., vol.28, no.5, pp.2192,2203, May 2013.

[16] Dan Wang; Chengxiong Mao; Jiming Lu; Shu Fan; Luonan Chen, "The Research on Characteristics of Electronic Power Transformer for Distribution System", IEEE/PES Transmission and Distribution Conference and Exhibition: Asia and Pacific, pp.1,5, 2005

[17] G. Ortiz, D. Bortis, J. W. Kolar, O. Apeldoorn, "Soft-Switching Techniques for Medium-Voltage Isolated Bidirectional DC/DC Converters in Solid State Transformers", Proceedings of the 38th Annual Conference of the IEEE Industrial Electronics Society (IECON 2012), Montreal, Canada, October 25-28, 2012

[18] N. Schibli, "Symmetrical Multilevel Converters with Two Quadrant DCDC Feeding", Ph.D. dissertation, Swiss Federal Institue of Technology Lausanne, 2000.

[19] Todorcevic, T.; Bauer, P.; Ferreira, J.A.; van Kessel, R., "Bidirectional modular multilevel DC-DC converter control and loss modeling for energy extraction from Electro Active Polymer Wave Energy generator" IEEE ECCE Asia Downunder (ECCE Asia), pp.461,467, 3-6 June 2013

[20] Haihua Zhou; Khambadkone, A.M., "Hybrid Modulation for DualActive-Bridge Bidirectional Converter With Extended Power Range for Ultracapacitor Application", IEEE Transactions on Industry Applications , vol.45, no.4, pp.1434,1442, July-aug. 2009. 